\title{
A note on the random greedy triangle-packing algorithm
}

\author{
Tom Bohman*, Alan Frieze ${ }^{\dagger}$ and Eyal Lubetzky
}

\begin{abstract}
The random greedy algorithm for constructing a partial SteinerTriple-System is defined as follows. We begin with a complete graph on $n$ vertices and proceed to remove the edges of triangles one at a time, where each triangle removed is chosen uniformly at random from the collection of all remaining triangles. This stochastic process terminates once it arrives at a triangle-free graph. In this note we show that with high probability the number of edges in the final graph is at most $n^{7 / 4+o(1)}$.
\end{abstract}

\section{Introduction}

We consider the random greedy algorithm for triangle-packing. This stochastic graph process begins with the graph $G(0)$, set to be the complete graph on vertex set $[n]$, then proceeds to repeatedly remove the edges of randomly chosen triangles (i.e. copies of $K_{3}$ ) from the graph. Namely, letting $G(i)$ denote the graph that remains after $i$ triangles have been removed, the $(i+1)$-th triangle removed is chosen uniformly at random from the set of all triangles in $G(i)$. The process terminates at a triangle-free graph $G(M)$. In this work we study the random variable $M$, i.e., the number of triangles removed until obtaining a triangle-free graph (or equivalently, how many edges there are in the final triangle-free graph).

This process and its variations play an important role in the history of combinatorics. Note that the collection of triangles removed in the course of the process is a maximal collection of 3 -element subsets of $[n]$ with the property that any pair of distinct triples in the collection have an intersection of cardinality less than 2. For integers $t<k<n$ a partial $(n, k, t)$-Steiner system is a collection of $k$-element subsets of an $n$-element set with the property that any pairwise intersection of sets in the collection has cardinality

arXiv: 1004.2418

*Research supported in part by NSF grants DMS-0701183 and DMS-1001638.

${ }^{\dagger}$ Research supported in part by NSF grant DMS-0721878. 
less than $t$. Note that the number of sets in a partial $(n, k, t)$-Steiner system is at most $\left(\begin{array}{c}n \\ t\end{array}\right) /\left(\begin{array}{l}k \\ t\end{array}\right)$. Let $S(n, k, t)$ be the maximum number of $k$-sets in a partial $(n, k, t)$-Steiner system. In the early 1960's Erdös and Hanani [5] conjectured that for any integers $t<k$

$$
\lim _{n \rightarrow \infty} \frac{S(n, k, t)\left(\begin{array}{l}
k \\
t
\end{array}\right)}{\left(\begin{array}{l}
n \\
t
\end{array}\right)}=1 .
$$

In words, for any $t<k$ there exist partial $(n, k, t)$-Steiner systems that are essentially as large as allowed by the simple volume upper bound. This conjecture was proved by Rödl [7] in the early 1980's by way of a randomized construction that is now known as the Rödl nibble. This construction is a semi-random variation on the random greedy triangle-packing process defined above, and thereafter such semi-random constructions have been successfully applied to establish various key results in Combinatorics over the last three decades (see e.g. [1] for further details).

Despite the success of the Rödl nibble, the limiting behavior of the random greedy packing process remains unknown, even in the special case of triangle packing considered here. Recall that $G(i)$ is the graph remaining after $i$ triangles have been removed. Let $E(i)$ be the edge set of $G(i)$. Note that $|E(i)|=\left(\begin{array}{l}n \\ 2\end{array}\right)-3 i$ and that $E(M)$ is the number of edges in the triangle-free graph produced by the process. Observe that if we show $|E(M)|=o\left(n^{2}\right)$ with non-vanishing probability then we will establish (1) for $k=3, t=2$ and obtain that the random greedy triangle-packing process produces an asymptotically optimal partial Steiner system. This is in fact the case: It was shown by Spencer [9] and independently by Rödl and Thoma [7] that $|E(M)|=o\left(n^{2}\right)$ with high probability ${ }^{1}$. This was extended to $|E(M)| \leq n^{11 / 6+o(1)}$ by Grable in [6], where the author further sketched how similar arguments using more delicate calculations should extend to a bound of $n^{7 / 4+o(1)}$ w.h.p.

By comparison, it is widely believed that the graph produced by the random greedy triangle-packing process behaves similarly to the Erdös-Rényi random graph with the same edge density, hence the process should end once its number of remaining edges becomes comparable to the number of triangles in the corresponding Erdős-Rényi random graph. At this critical edge density a positive fraction of the edges in the Erdős-Rényi graph are not contained in any triangle, and such edges in $G(i)$ clearly remain in the final graph.

\footnotetext{
${ }^{1}$ Here and in what follows, "with high probability" (w.h.p.) denotes a probability tending to 1 as $n \rightarrow \infty$.
} 
Conjecture (Folklore). With high probability $|E(M)|=n^{3 / 2+o(1)}$.

Joel Spencer has offered $\$ 200$ for a resolution of this question. The authors are not aware of any proof of a non-trivial lower bound on $|E(M)|$ in the literature.

In this note we apply the differential-equation method for dynamic concentration to achieve an upper bound on $E(M)$. In contrast to the aforementioned nibble-approach, whose application in this setting involves delicate calculations, our approach yields a short proof of the following best-known result:

Theorem 1. Consider the random greedy algorithm for triangle-packing on $n$ vertices. Let $M$ be the number of steps it takes the algorithm to terminate and let $E(M)$ be the edges of the resulting triangle-free graph. Then with high probability, $|E(M)|=O\left(n^{7 / 4} \log ^{5 / 4} n\right)$.

Wormald [11] also applied the differential-equation method to this problem, deriving an upper bound of $n^{2-\epsilon}$ on $|E(M)|$ for any $\epsilon<\epsilon_{0}=1 / 57$ while stating that "some non-trivial modification would be required to equal or better Grable's result." Indeed, in a companion paper we combine the methods introduced here with some other ideas (and a significantly more involved analysis) to improve the exponent of the upper bound on $|E(M)|$ to $2-\frac{1}{2 \sqrt{2}}+o(1)$. This follow-up work will appear in [3].

\section{Evolution of the process in detail}

As is usual for applications of the differential equations method, we begin by specifying the random variables that we track. Of course, our main interest is in the variable

$$
Q(i) \triangleq \# \text { of triangles in } G(i) .
$$

In order to track $Q(i)$ we also consider the co-degrees in the graph $G(i)$ :

$$
Y_{u, v}(i) \triangleq|\{x \in[n]: x u, x v \in E(i)\}|
$$

for all $\{u, v\} \in\left(\begin{array}{c}{[n]} \\ 2\end{array}\right)$. Our interest in $Y_{u, v}$ is motivated by the following observation: If the $(i+1)$-th triangle taken is $a b c$ then

$$
Q(i+1)-Q(i)=Y_{a, b}(i)+Y_{b, c}(i)+Y_{a, c}(i)-2 .
$$

Thus, bounds on $Y_{u, v}$ yield important information about the underlying process. We mention in passing that Grable's argument hinges on an analysis 
of an analogous set of variables. The main difference is that Grable's nibblebased proof uses very different methods to bound variations. (And Grable provides the full details only to the point where $n^{11 / 6+o(1)}$ edges remain.)

Now that we have identified our variables, we determine the continuous trajectories that they should follow. We establish a correspondence with continuous time by introducing a continuous variable $t$ and setting

$$
t=i / n^{2}
$$

(this is our time scaling). We expect the graph $G(i)$ to resemble a uniformly chosen graph with $n$ vertices and $\left(\begin{array}{l}n \\ 2\end{array}\right)-3 i$ edges, which in turn resembles the Erdős-Rényi graph $G_{n, p}$ with

$$
p=1-6 i / n^{2}=p(t)=1-6 t .
$$

(Note that we can view $p$ as either a continuous function of $t$ or as a function of the discrete variable $i$. We pass between these interpretations of $p$ without comment.) Following this intuition, we expect to have $Y_{u, v}(i) \approx p^{2} n$ and $Q(i) \approx p^{3} n^{3} / 6$. For ease of notation define

$$
y(t)=p^{2}(t), \quad q(t)=p^{3}(t) / 6 .
$$

We state our main result in terms of an error function that slowly grows as the process evolves. Define

$$
f(t)=5-30 \log (1-6 t)=5-30 \log p(t) .
$$

Our main result is the following:

Theorem 2. With high probability we have

$$
\begin{array}{lr}
Q(i) \geq q(t) n^{3}-\frac{f^{2}(t) n^{2} \log n}{p(t)} & \text { and } \\
\left|Y_{u, v}(i)-y(t) n\right| \leq f(t) \sqrt{n \log n} & \text { for all }\{u, v\} \in\left(\begin{array}{c}
{[n]} \\
2
\end{array}\right),
\end{array}
$$

holding for every

$$
i \leq i_{0}=\frac{1}{6} n^{2}-\frac{5}{3} n^{7 / 4} \log ^{5 / 4} n .
$$

Furthermore, for all $i=1, \ldots, M$ we have

$$
Q(i) \leq q(t) n^{3}+\frac{1}{3} n^{2} p(t) .
$$


Note that the error term in the upper bound (4) decreases as the process evolves. This is not a common feature of applications of the differential equations method for random graph process; indeed, the usual approach requires an error bound that grows as the process evolves. While novel techniques are introduced here to get this 'self-correcting' upper bound, two versions of 'self-correcting' estimates have appeared to date in applications of the differential equations method in the literature (see [4] and [10]). The stronger upper bound on the number of edges in the graph produced by the random greedy triangle-packing process given in the companion paper [3] is proved by establishing self-correcting estimates for a large collection of variables (including the variable $Y_{u, v}$ introduced here).

Observe that (2) (with $i=i_{0}$ ) establishes Theorem 1. We conclude this section with a discussion of the implications of (4) for the end of the process, the part of the process where there are fewer then $n^{3 / 2}$ edges remaining. Our first observation is that at any step $i$ we can deduce a lower bound on the number of edges in the final graph; in particular, for any $i$ we have $E(M) \geq E(i)-3 Q(i)$. We might hope to establish a lower bound on the number of edges remaining at the end of the process by showing that there is a step $i$ where $E(i)-3 Q(i)$ is large. The bound (4) is (just barely) too weak for this argument to be useful. But we can deduce the following. Consider $i=n^{2} / 6-\Theta\left(n^{3 / 2}\right)$; that is, consider $p=c n^{-1 / 2}$. Once $c$ is small enough the upper bound (4) is dominated by the 'error' term $n^{2} p / 3$. If $Q$ remains close to this upper bound then for the rest of the process we are usually just choosing triangles in which every edge is in exactly one triangle; in other words, the remaining graph is an approximate partial Steiner triple system. If $Q$ drops significantly below this bound then the process will soon terminate.

\section{Proof of Theorem 2}

The structure of the proof is as follows. For each variable of interest and each bound (meaning both upper and lower) we introduce a critical interval that has one extreme at the bound we are trying to maintain and the other extreme slightly closer to the expected trajectory (relative to the magnitude of the error bound in question). The length of this interval is generally a function of $t$. If a particular bound is violated then sometime in the process the variable would have to 'cross' this critical interval. To show that this event has low probability we introduce a collection of sequences of random variables, a sequence starting at each step $j$ of the process. This sequence stops as soon as the variable leaves the critical interval (which in many cases 
would be immediately), and the sequence forms either a submartingale or supermartingale (depending on the type of bound in question). The event that the bound in question is violated is contained in the event that there is an index $j$ for which the corresponding sub/super-martingale has a large deviation. Each of these large deviation events has very low probability, even in comparison with the number of such events. Theorem 2 then follows from the union bound.

For ease of notation we set

$$
i_{0}=\frac{1}{6} n^{2}-\frac{5}{3} n^{7 / 4} \log ^{5 / 4} n, \quad p_{0}=10 n^{-1 / 4} \log ^{5 / 4} n .
$$

Let the stopping time $T$ be the minimum of $M$ and the first step $i<i_{0}$ at which (2) or (3) fail and the first step $i$ at which (4) fails. Note that, since $Y_{u, v}$ decreases as the process evolves, if $i_{0} \leq i \leq T$ then we have

$$
Y_{u, v}(i)=O\left(n^{1 / 2} \log ^{5 / 2} n\right) \quad \text { for all }\{u, v\} \in\left(\begin{array}{c}
{[n]} \\
2
\end{array}\right) .
$$

We begin with the bounds on $Q(i)$. The first observation is that we can write the expected one-step change in $Q$ as a function of $Q$. To do this, we note that we have

$$
\mathbb{E}[\Delta Q \mid G(i)]=-\sum_{x y z \in Q} \frac{Y_{x y}+Y_{x z}+Y_{y z}-2}{Q}=2-\frac{1}{Q} \sum_{x y \in E} Y_{x y}^{2}
$$

and

$$
3 Q=\sum_{x y \in E} Y_{x y}
$$

(And, of course, $|E|=n^{2} p / 2-n / 2$.) Observe that if $Q$ grows too large relative to its expected trajectory then the expected change will be become more negative, introducing a drift to $Q$ that brings it back toward the mean. A similar phenomena occurs if $Q$ gets too small. Restricting our attention to a critical interval that is some distance from the expected trajectory allows us to take full advantage of this effect. This is the main idea in this analysis.

For the upper bound on $Q(i)$ our critical interval is

$$
\left(q(t) n^{3}+\frac{1}{4} n^{2} p, q(t) n^{3}+\frac{1}{3} n^{2} p\right) .
$$

Suppose $Q(i)$ falls in this interval. Since Cauchy-Schwartz gives

$$
\sum_{x y \in E} Y_{x y}^{2} \geq \frac{\left(\sum_{x y \in E} Y_{x y}\right)^{2}}{|E|} \geq \frac{9 Q^{2}}{n^{2} p / 2}
$$


in this situation we have

$$
\mathbb{E}[Q(i+1)-Q(i) \mid G(i)] \leq 2-\frac{18 Q}{n^{2} p}<2-3 n p^{2}-\frac{9}{2}=-3 n p^{2}-\frac{5}{2} .
$$

Now we consider a fixed index $j$. (We are interested in those indices $j$ where $Q(j)$ has just entered the critical window from below, but our analysis will formally apply to any $j$.) We define the sequences of random variables $X(j), X(j+1), \ldots, X\left(T_{j}\right)$ where

$$
X(i)=Q(i)-q n^{3}-\frac{n^{2} p}{3}
$$

and the stopping time $T_{j}$ is the minimum of $\max \{j, T\}$ and the smallest index $i \geq j$ such that $Q(i)$ is not in the critical interval (6). (Note that if $Q(j)$ is not in the critical interval then we have $T_{j}=j$.) In the event $j \leq i<T_{j}$ we have

$$
\begin{aligned}
\mathbb{E}[X(i+1)-X(i) \mid G(i)]= & \mathbb{E}[Q(i+1)-Q(i) \mid G(i)] \\
& -\left(q\left(t+1 / n^{2}\right)-q(t)\right) n^{3} \\
& -\left(p\left(t+1 / n^{2}\right)-p(t)\right) \frac{n^{2}}{3} \\
\leq & -3 n p^{2}-\frac{5}{2}+3 n p^{2}+2+O(1 / n) \\
\leq & 0 .
\end{aligned}
$$

So, our sequence of random variables is a supermartingale. Note that if $Q(i)$ crosses the upper boundary in (4) at $i=T$ then, since the one step change in $Q(i)$ is at most $3 n$, there exists a step $j$ such that

$$
X(j) \leq-\frac{n^{2} p(t(j))}{12}+O(n)
$$

while $T=T_{j}$ and $X(T) \geq 0$. We apply Hoeffding-Azuma to bound the probability of such an event. As our sequence of random variables starts at step $j$ of the process, the number of steps is at most $n^{2} p(t(j)) / 6$. The maximum 1-step difference is $O\left(n^{1 / 2} \log ^{5 / 2} n\right.$ ) (as $i<T$ implies bounds on the co-degrees). Thus the probability of such a large deviation beginning at step $j$ is at most

$$
\exp \left\{-\Omega\left(\frac{\left(n^{2} p(t(j))\right)^{2}}{\left(n^{2} p(t(j))\right) \cdot\left(n^{1 / 2} \log ^{5 / 2} n\right)^{2}}\right)\right\}=\exp \left\{-\Omega\left(\frac{n p(t(j))}{\log ^{5} n}\right)\right\} .
$$


As there are at most $n^{2}$ possible values of $j$, we have the desired bound.

Now we turn to the lower bound on $Q$, namely (2). Here we work with the critical interval

$$
\left(q(t) n^{3}-\frac{f(t)^{2} n^{2} \log n}{p}, q(t) n^{3}-\frac{(f(t)-1) f(t) n^{2} \log n}{p}\right) .
$$

Suppose $Q(i)$ falls in this interval for some $i<T$. Note that our desired inequality is in the wrong direction for an application of Cauchy Schwartz to (5). In its place we use the control imposed on $Y_{u, v}(i)$ by the condition $i<T$. For a fixed $3 Q=\sum_{u v \in E} Y_{u, v}$, the sum $\sum_{u v \in E} Y_{u, v}^{2}$ is maximized when we make as many terms as large as possible. Suppose this allows $\alpha$ terms in the $\operatorname{sum} \sum_{x y \in E} Y_{x y}$ equal to $n p^{2}+f \sqrt{n \log n}$ and $\alpha+\beta$ terms equal to $n p^{2}-f \sqrt{n \log n}$. For ease of notation we view $\alpha, \beta$ as rationals, thereby allowing the terms in the maximum sum to split completely into these two types. Then we have

$$
\beta f \sqrt{n \log n}=|E| \cdot n p^{2}-3 Q=3 q n^{3}-3 Q-\frac{n^{2} p^{2}}{2} .
$$

Therefore, we have

$$
\begin{aligned}
\sum_{x y \in E} Y_{x y}^{2} \leq & \alpha\left(n p^{2}+f \sqrt{n \log n}\right)^{2}+(\alpha+\beta)\left(n p^{2}-f \sqrt{n \log n}\right)^{2} \\
= & \left(\frac{n^{2} p}{2}-\frac{n}{2}\right) \cdot n^{2} p^{4}+\left(\frac{n^{2} p}{2}-\frac{n}{2}\right) \cdot f^{2} n \log n-2 \beta f p^{2} n^{3 / 2} \log ^{1 / 2} n \\
= & \frac{n^{4} p^{5}}{2}+\frac{f^{2} p n^{3} \log n}{2}-\frac{1}{2}\left(n^{3} p^{4}+f^{2} n^{2} \log n\right) \\
& -2 p^{2} n\left(3 q n^{3}-3 Q-\frac{n^{2} p^{2}}{2}\right) \\
\leq & 6 n p^{2} Q-\frac{n^{4} p^{5}}{2}+\frac{f^{2} p n^{3} \log n}{2}+\frac{n^{3} p^{4}}{2} .
\end{aligned}
$$

Now, for $j<i_{0}$ define $T_{j}$ to be the minimum of $i_{0}, \max \{j, T\}$ and the smallest index $i \geq j$ such that $Q(i)$ is not in the critical interval (7). Set

$$
X(i)=Q(i)-q(t) n^{3}+\frac{f(t)^{2} n^{2} \log n}{p(t)} .
$$


For $j \leq i<T_{j}$ we have the bound

$$
\begin{aligned}
\mathbb{E}[X & (i+1)-X(i) \mid G(i)] \\
= & \mathbb{E}[Q(i+1)-Q(i) \mid G(i)]-n^{3}\left(q\left(t+1 / n^{2}\right)-q(t)\right) \\
& +\left(\frac{f^{2}\left(t+1 / n^{2}\right)}{p\left(t+1 / n^{2}\right)}-\frac{f^{2}(t)}{p(t)}\right) n^{2} \log n \\
\geq & 2-6 n p^{2}+\frac{n^{4} p^{5}}{2 Q}-\frac{f^{2} p n^{3} \log n}{2 Q}+O(p)+3 p^{2} n+O(1 / n) \\
& +\left(\frac{2 f^{\prime} f}{p}+\frac{6 f^{2}}{p^{2}}\right) \log n+O\left(\frac{\log ^{3} n}{n^{2} p^{3}}\right) \\
\geq & \frac{(f-1) f n^{2} \log n}{p} \cdot \frac{n^{4} p^{5}}{2\left(q n^{3}\right)^{2}}-\frac{f^{2} p n^{3} \log n}{2 Q} \\
& +\left(\frac{2 f^{\prime} f}{p}+\frac{6 f^{2}}{p^{2}}\right) \log n \\
\geq & {\left[\frac{18 f^{2}}{p^{2}}-\frac{18 f}{p^{2}}-(1+o(1)) \frac{3 f^{2}}{p^{2}}+\left(\frac{2 f^{\prime} f}{p}+\frac{6 f^{2}}{p^{2}}\right)\right] \log n } \\
\geq & 0
\end{aligned}
$$

If the process violates the bound (2) at step $T=i$ then there exists a $j<i$ such that $T=T_{j}, X(T)=X(i)<0$ and

$$
X(j)>\frac{f(t(j)) n^{2} \log n}{p(t(j))}-O(n) .
$$

The submartingale $X(j), X(j+1), \ldots, X\left(T_{j}\right)$ has length at most $n^{2} p(t(j)) / 6$ and maximum one-step change $O\left(n^{1 / 2} \log ^{5 / 2} n\right)$. The probability that we violate the lower bound $(2)$ is at most

$$
\begin{gathered}
n^{2} \cdot \exp \left\{-\Omega\left(\frac{f^{2}(t(j)) n^{4} \log ^{2} n / p^{2}(t(j))}{n^{2} p(t(j)) \cdot n \log ^{5} n}\right)\right\} \\
=n^{2} \cdot \exp \left\{-\Omega\left(\frac{f^{2}(t(j)) n}{\log ^{3} n}\right)\right\}=o(1) .
\end{gathered}
$$

Finally, we turn to the co-degree estimate $Y_{u, v}$. Let $u, v$ be fixed. We begin with the upper bound. Our critical interval here is

$$
(y(t) n+(f(t)-5) \sqrt{n \log n}, y(t) n+f(t) \sqrt{n \log n}) .
$$


For a fixed $j<i_{0}$ we consider the sequence of random variables $Z_{u, v}(j)$, $Z_{u, v}(j+1), \ldots, Z_{u, v}\left(T_{j}\right)$ where

$$
Z_{u, v}(i)=Y_{u, v}(i)-y(t) n-f(t) \sqrt{n \log n}
$$

and $T_{j}$ is defined to be the minimum of $i_{0}, \max \{j, T\}$ and the smallest index $i \geq j$ such that $Y_{u, v}(i)$ is not in the critical interval (8). To see that this sequence forms a supermartingale, we note that $i<T$ gives

$$
\left|Q(i)-q(t) n^{3}\right| \leq \frac{f(t)^{2} n^{2} \log n}{p(t)},
$$

and therefore

$$
\begin{aligned}
\mathbb{E}[ & \left.Z_{u, v}(i+1)-Z_{u, v}(i)\right] \\
\leq & -\sum_{x \in N(u) \cap N(v)} \frac{Y_{u, x}+Y_{v, x}-1_{u v \in E(i)}}{Q} \\
& -n\left(y\left(t+1 / n^{2}\right)-y(t)\right)-\sqrt{n \log n}\left(f\left(t+1 / n^{2}\right)-f(t)\right) \\
\leq & -\frac{2(y n+(f-5) \sqrt{n \log n)(y n-f \sqrt{n \log n})}}{Q}+O\left(\frac{1}{n^{2} p}\right) \\
& -\frac{y^{\prime}(t)}{n}-f^{\prime}(t) \frac{\log ^{1 / 2} n}{n^{3 / 2}}+O\left(\frac{1}{n^{3} p^{2}}\right) \\
\leq & -\frac{2(y n+(f-5) \sqrt{n \log n})(y n-f \sqrt{n \log n})}{q n^{3}}+O\left(\frac{1}{n^{2} p}\right) \\
& +2 \cdot \frac{f^{2} n^{2} \log n}{p} \cdot \frac{(y n)^{2}}{\left(q n^{3}\right)^{2}}-\frac{y^{\prime}(t)}{n}-f^{\prime}(t) \frac{\log ^{1 / 2} n}{n^{3 / 2}} \\
\leq & \frac{10 y n^{3 / 2} \log ^{1 / 2} n}{q n^{3}}+\frac{14 f^{2} n \log n}{q n^{3}}+O\left(\frac{1}{n^{2} p}\right)-f^{\prime}(t) \frac{\log ^{1 / 2} n}{n^{3 / 2}}
\end{aligned}
$$

To get the supermartingale condition we consider each positive term here separately. The following bounds would suffice

$$
\frac{60}{p} \leq \frac{f^{\prime}}{3}, \quad \frac{84 f^{2} \sqrt{\log n}}{p^{3} n^{1 / 2}} \leq \frac{f^{\prime}}{3}, \quad \frac{1}{n^{1 / 2} p}=o\left(f^{\prime} \sqrt{\log n}\right) .
$$

The first term requires

$$
f^{\prime}(t) \geq \frac{180}{p(t)}=\frac{180}{1-6 t} .
$$


We see that this requirement, together with the initial condition $f(0) \geq 5$, imposes

$$
f(t) \geq 5-30 \log (1-6 t)=5-30 \log p(t) .
$$

But this value for $f$ also suffices to handle the remaining terms as we restrict our attention to $p \geq p_{0}=10 n^{-1 / 4} \log ^{5 / 4} n$. Thus, we have established that $Z_{u, v}(i)$ is a supermartingale.

To bound the probability of a large deviation we recall a Lemma 6 from [2]. A sequence of random variables $X_{0}, X_{1}, \ldots$ is $(\eta, N)$-bounded if for all $i$ we have

$$
-\eta<X_{i+1}-X_{i}<N
$$

Lemma 3. Suppose $0 \equiv X_{0}, X_{1}, \ldots$ is an $(\eta, N)$-bounded submartingale for some $\eta<N / 10$. Then for any $a<\eta m$ we have

$$
\mathbb{P}\left(X_{m}<-a\right)<\exp \left(-a^{2} /(3 \eta N m)\right) \text {. }
$$

As $-Z_{u, v}(j),-Z_{u, v}(j+1), \ldots$ is a $(6 / n, 2)$-bounded submartingale, the probability that we have $T=T_{j}$ with $Y_{u, v}(T)>y n+f \sqrt{n \log n}$ is at most

$$
\exp \left\{-\frac{25 n \log n}{3 \cdot(6 / n) \cdot 2 \cdot\left(p(t(j)) n^{2} / 6\right)}\right\} \leq \exp \left\{-\frac{25 \log n}{6}\right\} \text {. }
$$

Note that there are at most $n^{4}$ choices for $j$ and the pair $u, v$. As the argument for the lower bound in (3) is the symmetric analogue of the reasoning we have just completed, Theorem 2 follows.

\section{Acknowledgements}

The authors thank the anonymous referees for their helpful comments.

\section{References}

[1] N. Alon, J.H. Spencer, The Probabilistic Method (3rd Ed.), WileyInterscience, 2008. MR2437651

[2] T. Bohman, The triangle-free process. Advances in Mathematics 221 (2009) 1653-1677. MR2522430

[3] T. Bohman, A. Frieze, E. Lubetzky, Random greedy triangle-packing beyond the $7 / 4$ barrier. In preparation. 
[4] T. Bohman, M. Picollelli, Evolution of SIR epidemics on random graphs with a fixed degree sequence. Manuscript.

[5] P. Erdős, H. Hanani, On a limit theorem in combinatorial analysis. Publicationes Mathematicae Debrecen 10 (1963) 10-13. MR0166116

[6] D. Grable, On random greedy triangle packing. Electronic Journal of Combinatorics 4 (1997), R11, 19 pp. MR1445383

[7] V. Rödl, On a packing and covering problem. European Journal of Combinatorics 6 (1985) 69-78. MR0793489

[8] V. Rödl, L. Thoma, Asymptotic packing and the random greedy algorithm. Random Structures and Algorithms 8 (1996) 161-177. MR1605397

[9] J.H. Spencer, Asymptotic packing via a branching process. Random Structures and Algorithms 7 (1995) 167-172. MR1369062

[10] A. Telcs, N.C. Wormald, S. Zhou, Hamiltonicity of random graphs produced by 2-processes. Random Structures and Algorithms 31 (2007) 450-481. MR2362639

[11] N.C. Wormald, The differential equation method for random graph processes and greedy algorithms, in Lectures on Approximation and Randomized Algorithms (M. Karonski and H.J. Prömel, eds), pp. 73-155. PWN, Warsaw, 1999.

TOM BOHMAN

Department of Mathematical Sciences

Carnegie Mellon University

Pittsburgh, PA 15213, USA

E-mail address: tbohman@math.cmu.edu

Alan Frieze

Department of Mathematical Sciences

Carnegie Mellon University

Pittsburgh, PA 15213, USA

E-mail address: alan@random.math.cmu.edu

EYAL LUBETZKY

Microsoft Research

One Microsoft WAY

REDMOND, WA 98052, USA

E-mail address: eyal@microsoft.com

Received April 13, 2010 